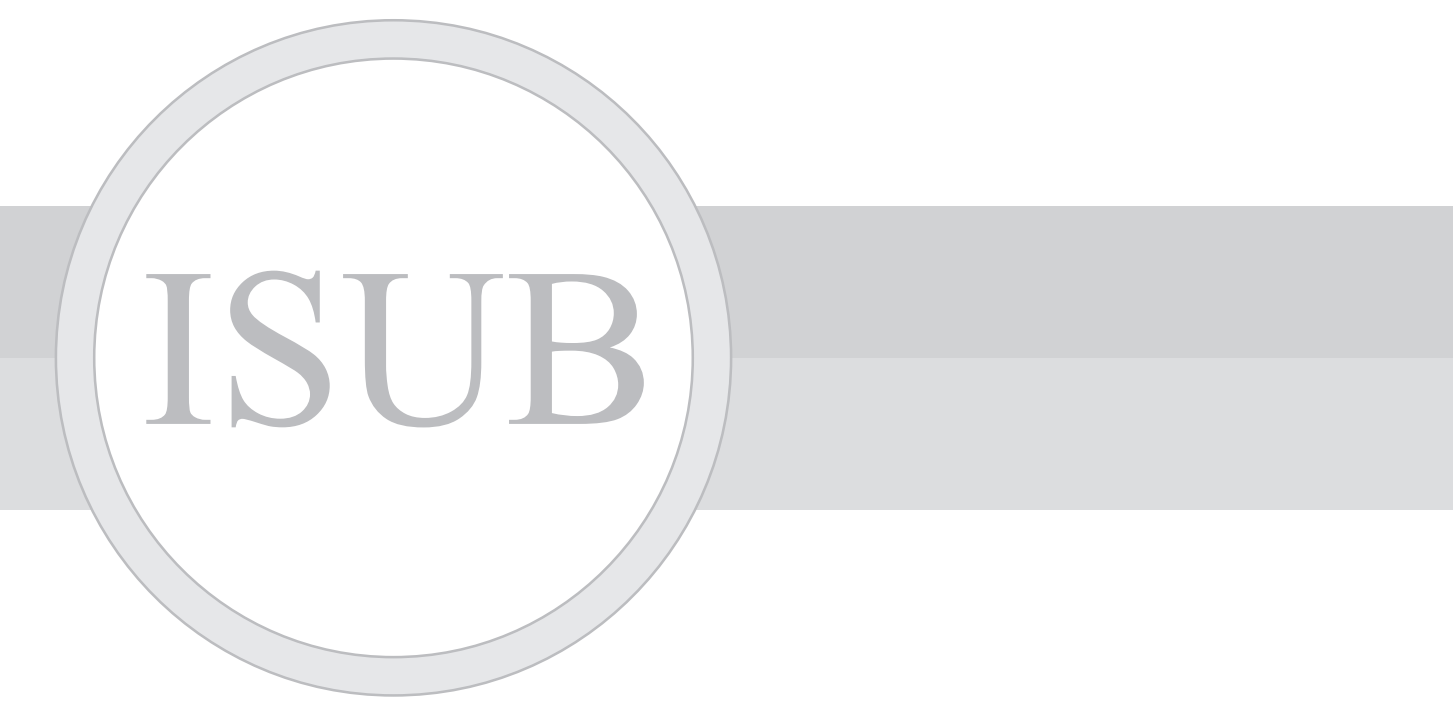

Enero - Junio de 2018 Vol. 5 Nro. 1

La Revista Investigación en Salud Universidad de Boyacá, está indizada a texto completo en:

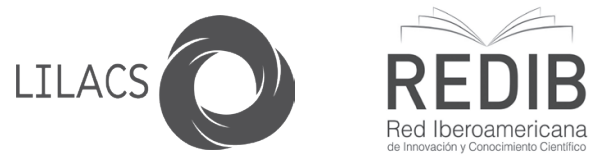


Revista Investigación en Salud Universidad de Boyacá, Facultad de Ciencias de la Salud, Universidad de Boyacá

Vol. 5 Nro. 1 Enero - Junio 2018 - Tunja, Colombia

ISSN: $2389-7325$

ISSN: 2539-2018 (En línea)

Periodicidad Semestral - Ediciones Universidad de Boyacá

\section{POLÍTICA EdITORIAL}

La Revista Investigación en Salud de la Universidad de Boyacá (ISUB), es una revista científica de publicación semestral arbitrada (modalidad doble ciego), la cual se encarga de publicar artículos científicos derivados de trabajos de investigación y/o académicos Nacionales e Internacionales con el fin de contribuir a aportar conocimientos del área de la salud, para la formación y actualización científica de sus lectores.

\section{Público Objetivo}

La Revista está dirigida a profesionales, investigadores, docentes y estudiantes de Ciencias de la Salud.

\section{Propiedad INTELECTUAL}

Los autores ceden a la Revista Investigación en Salud de la Universidad de Boyacá, los derechos de autor y propiedad intelectual, derechos de copia en todas las formas y medios conocidos. En caso de no ser publicado el artículo, la Universidad de Boyacá accede a retornar los derechos enunciados a los autores. La evidencia científica, el conflicto de intereses y el contenido de los documentos son de responsabilidad exclusiva y única de los autores.

\section{DiRECTIVOS UnIVERSIDAD DE BOYACÁ}

Presidente Universidad de Boyacá

Dr. Osmar Correal Cabral

\section{Rectora}

Dra. Rosita Cuervo Payeras

Vicerrector Académico

Ing. MSc. Rodrigo Correal Cuervo

Vicerrector Desarrollo Institucional Ing. MSc. Andrés Correal Cuervo

Vicerrectora Investigación, Ciencia y Tecnología Ing. Mg. Claudia Patricia Quevedo Vargas

Vicerrectora Educación Virtual Ing. Mg. Carmenza Montañez Torres

Vicerrector Administrativo y de Infraestructura Dr. Camilo Correal C.

\section{Editora}

Mg. Sandra Helena Suescún Carrero

\section{SOlicitud de CANJe}

Politeca - Universidad de Boyacá

Carrera 2a. Este Nro. 64 - 169

Teléfono: +578 7452105 Ext. 7300

Correo: politeca@uniboyaca.edu.co

Tunja, Boyacá - Colombia 


\section{INFORMACIÓN Y CORRESPONDENCIA}

Facultad de Ciencias de la Salud Universidad de Boyacá

Correo: revcis@uniboyaca.edu.co

Télefono: +57 87452742 Ext. 1107

http://revistasdigitales.uniboyaca.edu.co/index.php/rs/ issue/archive

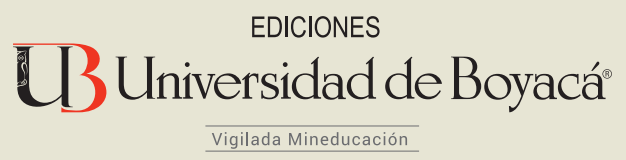

Carrera 2a. Este Nro. 64 - 169

Teléfono: +57 87452105 Ext: 3104

Correo: publicaciones@uniboyaca.edu.co

Tunja, Boyacá - Colombia

\section{Comité Editorial / Científico}

MSc. Rubén Darío Restrepo

Universidad de Texas, USA

F.T. Ph.D. Andrea Vásquez Morales

Universidad Manuela Beltrán, Colombia

MD. Gloria Eugenia Camargo Villalba

Universidad de Boyacá, Colombia

Bac. MSc. Luis Eduardo Traviezo Valles

UCLA, Venezuela

MD. Ph.D. Nicolás Ottone

Universidad de La Frontera, Temuco, Chile

Biol. Ph.D. Orlando Torres

Instituto Nacional de Salud, Colombia

Biol. Ph.D. Jorge Eduardo Duque

Universidad de Caldas, Colombia

Mg. Carolina Sandoval Cuellar

Universidad de Boyacá, Colombia

Msc. Luis Ernesto Ballesteros Acuña

Universidad Industrial de Santander, Colombia

Ph.D. Héctor Pons

Universidad del Zulia, Venezuela
Biol. Ph.D. Jazmir Quiroz

Instituto Venezolano de Investigaciones Clentíficas IVI, Venezuela

OD. Mg. Martha Inés Bernal García

Universidad de Boyacá, Colombia

Enf. Ph.D. Sonia Patricia Carreño Moreno Universidad Nacional, Colombia

Enf. Ph.D. Lina María Vargas

Universidad Nacional, Colombia

Enf. Ph.D. Olivia Lorena Chaparro Díaz

Universidad Nacional, Colombia

Biol. MSc. Atilio Junior Ferrebuz Cardozo

Universidad de Boyacá, Colombia

MD. Ph.D. Mariano Guillermo del Sol

Universidad de Temuco Chile

MD. Ph.D. Gustavo Otegui

Universidad de Buenos Aires, Argentina

Enf. Msc. Mónica Paola Quemba Mesa

Universidad de Boyacá, Colombia

Biol. Ph.D. Cristiane Angélica Ottoni

Universidad estadual paulista "Julio de Mesquita Fillio", Brasi

Biol. Ph.D. Patricia Locosque

Fundación Parque Zoológico de Sao Paulo, Brasil

ND. Dra. HC. Yolanda Torres de Galvis

Universidad CES-Universidad el Rosario, Colombia

MD. PHD. Julio César Carrero

Universidad Nacional Autónoma de México

Mic. Ph. D. Nuri Andrea Merchán

Universidad de Boyacá, Colombia

FT. Ph.D. Dernival Bertoncello

Universidad Federal do Triángulo Minero

MD. Ph.D. María Isabel Miguel Pérez

Universidad de Barcelona 
Corrección de texto y estilo

MD, MPH Carlos Arturo Hernández

Traducción al Inglés

MSc. Atilio Junior Ferrebuz Cardozo

Traducción al Portugués

Ph. D. Nuri Andrea Merchán

Diseño y Diagramación

División de Publicaciones

Impresión

Búhos Editores Ltda.

Número de ejemplares: 200

Tunja - Boyacá - Colombia

Colaboradores

Ph.D. Consuelo Vélez

Universidad de Caldas

Ph.D. Paulina Beverido Sustaeta

Universidad Veracruzana. México

Ph.D. María Cristina Ortiz León

Universidad Veracruzana. México

MSc. Antonio José Sánchez Serrano

Centro de investigaciones oncológicas San Diego

Ph.D. Jaime Andres Torres Ortiz

Universidad Pedagógica y Tecnológica de Colombia

Msc. Diana Georgina García Lozada

Universidad del Bosque

Ph.D. Dabeiba Adriana García Robayo

Pontificia Universidad Javeriana

Ph.D. Doris Rodríguez Leal

Universidad del Tolima
Ph.D. Juan Guillermo Díaz Bernal

Universidade Federal de Uberlândia

Mg. Javier Martínez Torres

Universidad Santo Tomás, Colombia

Mg. Diana Milena Díaz Vidal

Universidad San Buenaventura de Cartagena

Ph.D. Roy José Andrade Becerra

Universidad Pedagógica y Tecnológica de Colombia

Ph.D. Bruno Lamonte

Universidad de Costa Rica

MD. José Luis Bustos Sánchez

Universidad Pedagógica y Tecnológica de Colombia

MSc. Diego José García Corredor

Universidad Pedagógica y Tecnológica de Colombia

MV. MSc. Germán Augusto Baquero Sastre

Universidad Manuela Beltrán

Mg. Margareth Alfonso

Universidad de la Sabana

Ph.D. Gloria Carvajal Carrascal

Universidad de la Sabana

Mg. Diana di Filippo Villa

Universidad de Antioquia

Mg. Alexandra Porras

Universidad del Bosque

Mg. Guillermo Adrián Rivera Cardona

Pontificia Universidad Javeriana, Sede Cali

Mg. Norton Pérez Gutiérrez

Universidad Cooperativa de Colombia

Mg. Lu An González Santiago

Universidad Distrital, Colombia 
MSc. Kristian Andrés Espinosa Garnica Instituto de Evaluación Tecnológica en Salud (IETS), Colombia

Mg. Anyela Mancilla Lucumi Hospital Universitario de la Samaritana, Colombia

Ph.D (c) Anderson Rocha-Buelvas

Centro de Estudios en Salud de Universidad de Nariño (CESUN), Colombia

Mg. Alexandra López López

Universidad Autónoma de Manizales, Colombia

MSc. Angela Mayerly Cubides Munevar

Universidad del Valle - Universidad Libre -

Fundación Universitaria San Martin, Colombia

Ph.D. Giovane Mendieta Izquierdo

Universidad Militar, Colombia

MSc. Edgar Drebay Hernández Álvarez

Universidad Nacional de Colombia, Colombia

Ph. D Jaime Andrés Torres Ortiz

Universidad Pedagógica y Tecnológica de Colombia

Mg. Alexandra López López

Universidad Autónoma de Manizales, Colombia

MSc. Angela Mayerly Cubides Munevar

Universidad del Valle - Universidad Libre - Fundación

Universitaria San Martin, Colombia

Ph. D Jorge Enrique Correa Bautista

Universidad del Rosario, Colombia

Mg. Adriana Lucia Castellanos Garrido

Universidad de la Sabana, Colombia

Esp. Esther Cecilia Wilches Luna

Universidad del Valle, Colombia
MSc Marin Alonso Beyond Rojas

Universidad de Ciencias Aplicadas y Ambientales U.D.C.A, Colombia

Ph. D Linda Patricia Rocha Muñoz

Universidad Santo Tomás seccional Bucaramanga, Colombia

Ph. D Martha Lucia Díaz Galvis

Universidad Industrial de Santander, Colombia 


\section{CONTENIDO/CONTENT/CONTEÚDO}

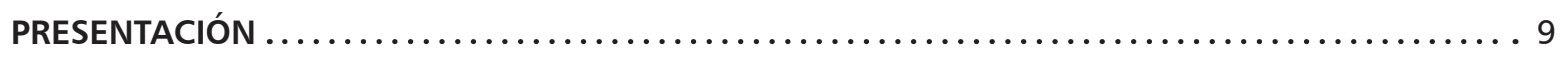

\section{EDITORIAL}

La investigación científica, ideología causal en ciencias de la salud

Martha Inés Bernal- García

\section{ARTICULOS DE INVESTIGACIÓN / RESEARCH ARTICLES / ARTIGOS DE PESQUISA}

Determinación de hemólisis en cepas de Staphylococcus spp.

causantes de mastitis bovina

Determination of hemolysis in Staphylococcus spp. strains which cause bovine mastitis

Determinação de hemólise em cepas de Staphylococcus spp. que causam mastite bovina

Yaline Sánchez-Neira, Maritza Angarita-Merchán

Seroprevalencia de infección por Trypanosoma cruzi y factores asociados, en Miraflores, Boyacá, Colombia

Seroprevalence of Trypanosoma cruzi infection and associated factors in Miraflores, Boyacá, Colombia

Soroprevalência da infecção por Trypanosoma cruzi e fatores associados, em Miraflores, Boyacá, Colômbia

Ángela Monroy-Díaz, Sonia Rodríguez-Niño, Sandra Helena Suescún-Carrero, Laura Ramírez-López

Percepción de la calidad del servicio en un centro de fisioterapia de una institución universitaria 
Perception of service quality at the physiotherapy center of a university institution

Percepção da qualidade do serviço em um centro de fisioterapia de uma instituição universitária

Elisa Andrea Cobo-Mejía, Leidy Yohana Archila-León, Estefanía Gutiérrez-Barrera, Ibeth

Dayanna Araque-Sepúlveda

Carga de la enfermedad de Chagas en el departamento de Boyacá en los años 2014 a 2016

Burden of Chagas' disease in Boyacá in the years 2014-2016

Carga de doença de Chagas no departamento de Boyacá nos anos de 2014

a 2016

Karen Natalia Gutiérrez-Daza, Karen Nayive Roa-Cortés, Yesenia María Ávila-Peña, Adriana

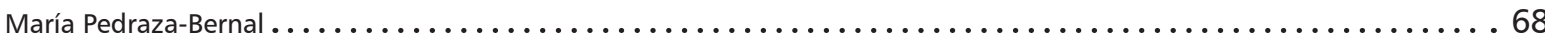

Nivel de sobrecarga en cuidadores informales de personas en situación de discapacidad cognitiva de Duitama, estudio exploratorio

Level of overloading in informal caregivers of people in situation of cognitive disability of Duitama, exploratory study

Nível de sobrecarga em cuidadores informais de pessoas em situação de deficiência cognitiva na cidade de Duitama na Colômbia,

estudo exploratório

Yesmith Rodríguez-Albañil, Mónica Paola Quemba-Mesa

Prevalencia de la sintomatología respiratoria en trabajadores mineros del municipio de Socotá, Boyacá, 2017

Prevalence of respiratory symptomatology in miners in the municipality of Socotá, Boyacá, 2017 
Prevalência de sintomas respiratórios em trabalhadores da mineração no município de Socotá, Boyacá, 2017

Nelson Camilo Gutiérrez-Alvarado, Yuli Velandia-Cristiano, Lady Hernández-Mongui,

Juliet Vargas-Neiza.

Caracterización fenotípica de cepas de Staphylococcus aureus productoras de B-lactamasas y resistente a la meticilina

Phenotypic characterization of Staphylococcus aureus strains that produce B-lactamases and are resistant to methicillin

Caracterização fenotípica de cepas de Staphylococcus aureus produtoras de B-lactamases e resistentes à meticilina

Yaline Sánchez, Eliana Ximena Urbano, Fernando José González, Atilio Junior Ferrebuz .................125

\section{CARTA AL EDITOR / LETTER TO THE EDITOR / CARTA AO EDITOR}

Trofozoíto de Entamoeba coli con cuatro núcleos

Entamoeba coli trophozoite with four nuclei

Trofozoíto de Entamoeba coli com quatro núcleos

Luis EduardoTraviezo, Andrea Galíndez .144

INDICACIONES PARA LOS AUTORES 149 INDICATIONS TO AUTHORS

INSTRUÇÕES AOS AUTORES

ÍNDICE DE ARTÍCULOS PUBLICADOS (2014-2017) INDEX OF ARTICLES PUBLISHED (2014-2017)

ÍNDICE DE ARTIGOS POSTADOS (2014-2017) 


\section{PRESENTACIÓN}

La profesora Martha Bernal García, profesora titular del programa de Medicina de la Facultad d Ciencias de la salud de la Universidad de Boyacá, en su nota editorial aborda el tema de la investigación científica como una ideología causal en ciencia de la salud, dando a entender que hay diferentes aproximaciones epistemológicas para delimitar el proceso de generar nuevos conocimientos, para obtener respuestas a los problemas y necesidades no resueltas del ser humano.

Plantea varios interrogantes válidos en el análisis de si todas las investigaciones deben considerarse científicas y si las prácticas de las habilidades clínicas general conocimiento científico.

La Facultad de ciencias de la salud presenta en este nuevo número de su revista investigación en salud, los resultados de varias investigaciones que tiene una singular importancia por la incidencia que tienen en la salud y bienestar de las personas de una determinada región o de una determinada actividad.

Particularmente es muy interesante indagar sobre problemas que sigue generando la enfermedad de Chagas en el departamento de Boyacá, especialmente en regiones en regiones donde todavía hay una gran prevalencia por efecto de la existencia de los comúnmente llamados "pito" que es el insecto hemíptero que transmite la enfermedad, también conocida como tripanosomiasis tropical, insectos que se desarrollan especialmente en los techos pajizos de muchas casas del campo boyacense.

Seguramente la lectura de los interesantes artículos contenidos en esta revista generará valiosos comentarios y servirá para proseguir en la búsqueda de nuevos conocimientos y la adopción de nuevasprácticas clínicas.

Osmar Correal Cabral

Presidente Consejo Directivo

Universidad de Boyacá 